\title{
Polymer brushes and their possible applications in artificial cilia research (Review)
}

\author{
JIAN ZHU，XUEMEI JIANG，JUN ZHONG and YIYUAN DUAN \\ Key Laboratory of Biorheological Science and Technology, Ministry of Education, \\ Bioengineering College, Chongqing University, Chongqing 400044, P.R. China
}

Received February 5, 2016; Accepted February 20, 2017

DOI: $10.3892 / \mathrm{mmr} .2017 .6533$

\begin{abstract}
The review aimed to assess the current research status of polymer brushes and artificial cilia, based on the analysis of polymer brush properties and the structure and function of natural cilia. The application of polymer brushes to develop artificial cilia has attracted increasing research interest, with rheological analysis often employed as an entry point for polymer mechanics. The present review assessed whether combing polymer brushes with artificial cilia, along with appropriate designing of high-polymer materials and use of suitable equipment, may to the development of a polymer net-structured material to imitate the morphology and functionality of a natural cilium system.
\end{abstract}

\section{Contents}

1. Introduction

2. Properties and research of stimulus-response polymer brushes

3. Structure and function of natural cilia and current research of artificial cilia

4. Application of polymer brushes in the field of artificial cilia

\section{Introduction}

Artificial trachea, a replacement procedure following tracheal overcut to repair tracheal defects (1), has overcome many complications frequent in earlier clinical trials (2), and exhibits great biocompatibility and structural stability (3). However, studies report that artificial trachea is linked to severe infections and restenosis due to the lack of cilia; the

Correspondence to: Dr Xuemei Jiang, Key Laboratory of Biorheological Science and Technology, Ministry of Education, Bioengineering College, Chongqing University, 174 Shazhengjie Street, Chongqing 400044, P.R. China

E-mail: jiangxuemei@cqu.edu.cn

Key words: polymer brushes, artificial cilia, application possibility airway waste is accumulated on the nude inner surface of the trachea where bacteria thrives, and the smooth inner surface of the trachea causes the surrounding tissues to grow inwards (4). To solve this issue, researchers have experimented with various parameters, for instance, altering the design of the internal diameter of transplants (5), as well as altering the surrounding microenvironment with anti-inflammatory factors (6). However, these findings require further validation. Recent developments in biomedical engineering and polymer science, including using polymer brushes as a method for surface modification, has led to further investigation into tracheal re-epithelization. Combining the preparation and molding technology of polymer materials with artificial cilia research, it is possible to produce a cilia-like film to coat the inner surface of a reconstructed airway to replace the natural damaged cilia, in order to remove airway waste. This strategy represents a potentially positive clinical application in the field of artificial cilia research.

\section{Properties and research of stimulus-response polymer brushes}

A polymer brush is a polymer system consisting of a high density of short chains grafted onto a surface to form a special topological structure, which appears as a stretched conformation. It resembles a brush between the short and main chains, influenced by a steric effect. Along with the rapid development of polymer science, the structure and length of polymer brush molecules may be controlled precisely; therefore, it presents an attractive applicability in many fields, including drug delivery $(7,8)$, lubrication $(9,10)$, altering the conductivity of a material's surface $(11,12)$, and controlling the transport of small particles $(13,14)$. In recent years, investigation into the stimulus-response polymer brush has involved biochemical, environmental biology and biomedical applications. This is owing to its ability to reversibly switch between adaptation and responsiveness via external stimulation, including temperature (15-17), $\mathrm{pH}$ value (18), ionic strength of the solvent $(19,20)$ and light (21). A schematic diagram of polymer brushes is shown in Fig. 1.

Temperature-responsive polymer brushes. Temperatureresponsive polymer brushes (17) can be divided into two types according to their functional groups: lower critical 
solution temperature (LCST) or upper critical solution temperature (UCST) group, including the polymers PNIPAM, PDMAEMA, PMEMA and PPO. Among them, PNIPAM is a typical temperature-sensitive polymer that is most frequently studied, and its height gradually as temperature increases. The responsiveness of temperature-responsive polymer brushes is affected by additional factors, including molecular weight and hydrophobicity of the monomer.

pH-responsive polymer brushes. The height of $\mathrm{pH}$-responsive polymer brushes (18) alters with environmental $\mathrm{pH}$ value, leading to the receipt or release of protons due to the ionizable groups in some polymers, including PAA, PMA, PDMAEMA and P4VP. This response relies on different properties of polymers; for example, the electrostatic repulsion between polymer chains or an alteration of solubility.

Solvent-responsive polymer brushes. Block polymer brushes or mixed polymer brushes are used to manufacture solvent-responsive polymer brushes (19) generally, and a morphological alteration is produced via phase separation to varying degrees in different solvents. This is common for the polymers PMMA, PBMA and PS. The morphological alteration of the polymer brush is obtained by altering the wettability through solvents; however, it may be influenced by other factors, including the relative length of all the blocks in the polymer chain.

Ionic strength-responsive polymer brushes. The ionic strength (20) is controlled to directly affect the electrostatic repulsion and steric effects of the polymer brush, and then the inner osmotic pressure and elasticity of molecular chain structure are affected immediately to alter the morphology of brushes. It has been demonstrated that the response of ionic strength-responsive polymer brushes is influenced by the concentration of salt (21), ionic radius (22) and the structure of the molecular chain (23).

Currently, polymer brushes are primarily used for surface modification, including alterations in wettability, permeability, absorbability and adhesion in other materials due to its unique topological structure (24). The modification ability is based on the strong electronic repulsion, entropic stretching costs and restricted constraints for irreversible grafting (25). For monomer polymer brush, the responsive activity relies on the density of grafts and the property of its functional groups, but for block polymer brush, the responsive activity relies on phase separation of different blocks and response to the relative reagent (26).

However, in the biological application of polymer brushes, there is a requirement to focus on the obtained conformation response by external stimuli and the controllability of the property of the material itself, as it is necessary to consider the long-term stabilization (physical and chemical) of the polymer material in vivo. The complicated environment of an organism renders it vital to develop a responsive system to control multiple stimuli efficiently.

\section{Structure and function of natural cilia and current research of artificial cilia}

Cilia is a type of hair-like organelle based on microtubule structures. Cilia are critical for movements of liquid through the body, but may also function as a cell receptor (27). They serve an important role in mediating bodily functions, alongside other hair-like structures. For example, the atrophy and loss of olfactory hairs on the top of nasopharyngeal epithelial tissue may cause anosphrasia (28). Auditory hairs serve a pivotal role in allowing a rapid hearing response to the vibration signals of sound waves, and grow on the inner ear hair cells; the microvilli of intestinal mucosal epithelia cells are an important structure for the body to assimilate nutrients; the fertilization process of sperms and eggs may only be accomplished with the help of the cilia in the human genital tract (29). Previous research has demonstrated that whether the cilia have motor activity primarily depends on the differences in ciliary axonemes (29). The ciliary structure is presented in Fig. 2 (30).

The present review primarily focused on active cilia. The cilia in airways eliminate waste via asymmetric and periodic bends to keep the airway clean and smooth. The movement of natural cilia uses the ATP stored in the cell body as energy to fuel the function of axonemal dynein. Previous studies indicated that, due to its small size, the Reynolds number of ciliary beat is less than $10^{-2}$, which conforms to the low Reynolds number conditions $(31,32)$. Therefore, the inertial effects are not significant and the flow relies on the liquid viscosity more.

Based on the structure and function of natural cilia, studies on artificial cilia have seen a rapid development in the last decade, especially in the field of lab-on-chip devices. Usually, these cilia are made of polymer materials prior to modification, and are used to manipulate fluid flow under certain external stimuli, including electrical $(33,34)$, magnetic $(32,35-37)$, light $(38,39), \mathrm{pH}(40-42)$ and temperature $(43,44)$. Overall, these developments of artificial cilia are closely associated with polymer science research, because polymers may be easily modified to functional material. Combined with proper design and assembly, polymers may reach adaptive responses easily for environmental temperature, humidity, atmosphere and micro stress. One of the first examples of artificial cilia to control fluid flow was an electric-actuated cilium (33). From a practical point of view, the electric-actuated cilia had three main limitations. The relatively high electric field extends into the fluid domain and therefore electro hydrodynamic effects may occur, which potentially leads to electrolysis. In addition, the principle did not work in conductive fluids, including certain biological fluids, because the electrical field may damage biological components of cells. Therefore, magnetic-actuated cilia have been developed to overcome these three primary limitations, and have become the most studied artificial cilia (45-47). They are made from a polymer matrix film consisting of embedded magnetic nanoparticles in response to the external magnetic field (48). In addition, other magnetic-actuated cilia have been synthesized using self-assembling super paramagnetic beads in an external magnetic field (32). Novel studies involving artificial cilia are introduced as follows.

Light-actuated cilia. Light-actuated artificial cilia (Fig. 3), have been developed from a polymer material consisting of light-activated chemical groups that may alter their conformation reversibly under different wavelengths of light, in order to simulate the oscillation motion of cilia (38). 
A

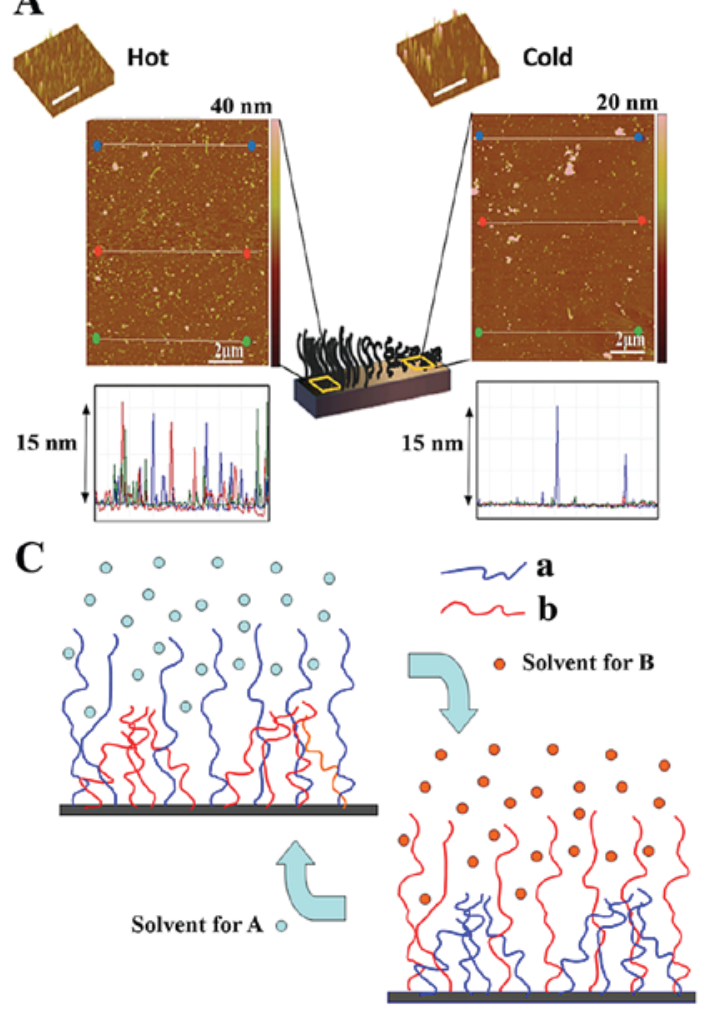

B
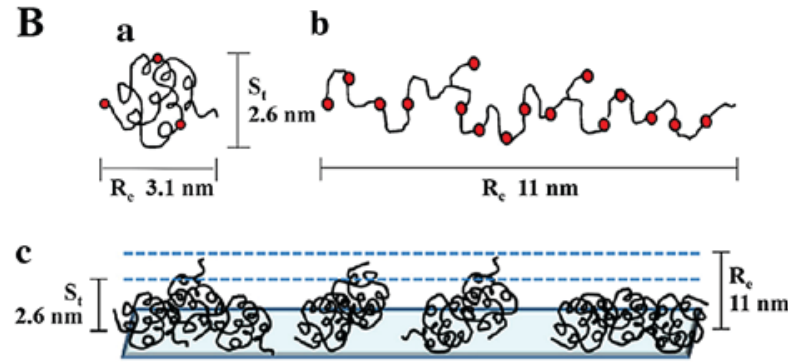

d

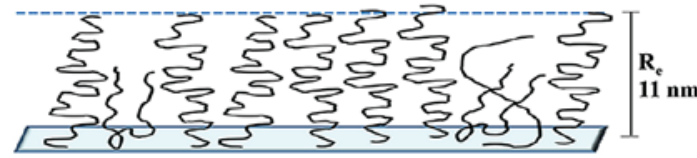

D

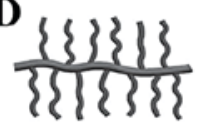

Figure 1. Schematic diagrams of polymer brushes. (A) Thermo-responsive polymer brush. Reproduced with permission (17). Atomic force microscope images (with corresponding 3D images at the top left corners) and 3 representative height profiles (below) taken from different areas of the 2-bromo-2-methyl-N-3 -(triethoxysilyl) propyl propanamide (BTPAm) layer after heating the silane coated $\mathrm{Si}$ substrate $\left(120-130^{\circ} \mathrm{C}, 20 \mathrm{~min}\right)$. Left: images and profiles taken from the hot side of the silicon wafer. Right: images and profile taken from the cold side. (B) pH-responsive polymer brush. Reproduced with permission (18). The upper pictures show the schematic diagrams of the $\mathrm{pH}$-responsive conformational change of linear polyethyleneimine (LP) with the red circles representing the charged units: (a) $\mathrm{pH} 7$ and (b) $\mathrm{pH}$ 3. The lower pictures show the schematic representations of LP molecules adsorbed on dicalcium phosphate dehydrate surfaces at (c) pH 7 and (d) pH 3. (C) Solvent-responsive polymer brush. Reproduced with permission (19). Schematic representation of solvent-responsive behavior for binary polymer brushes. (D) Ionic strength-responsive polymer brush. Reproduced with permission (20). Schematic representation of ionic strength-responsive behavior for cationic bottle-brush polymers.

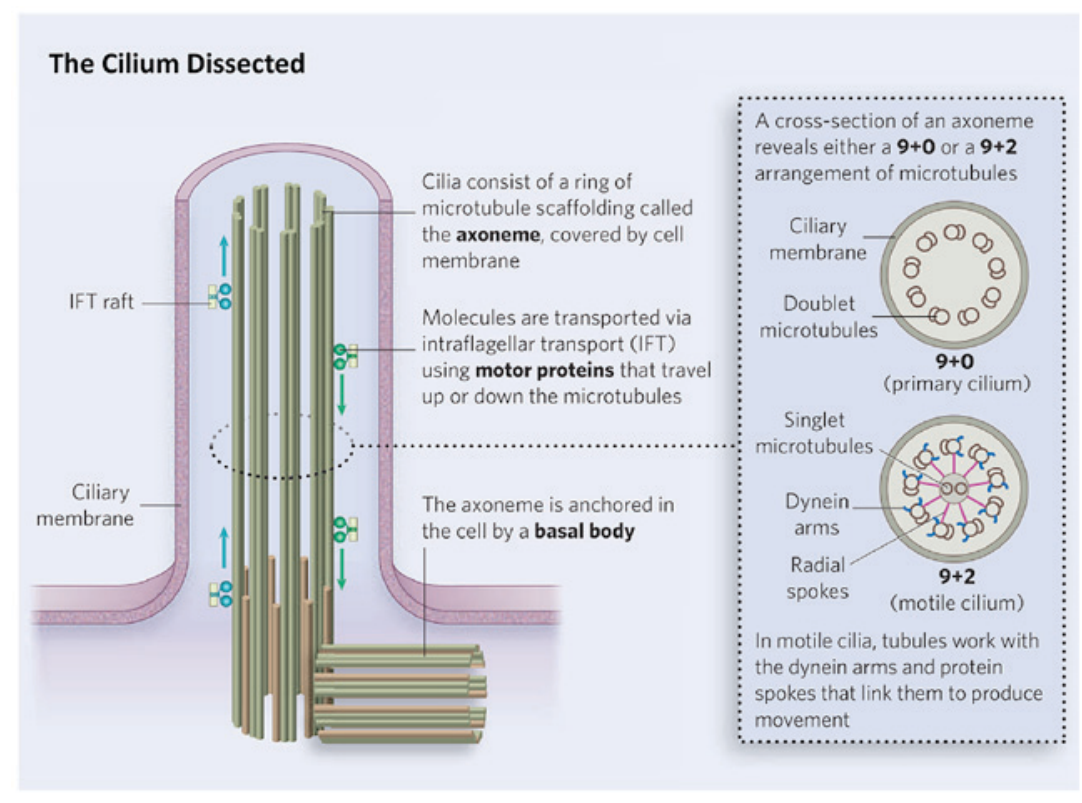

Figure 2. Diagram of natural ciliary structure. Reproduced with permission (23).

A new study from the University of Kiel (Kiel, Germany) demonstrated that new light sensitive artificial cilia have been developed to mount azobenzenes onto the cell membrane (39). The artificial cilia exhibited directional movement. The 
A

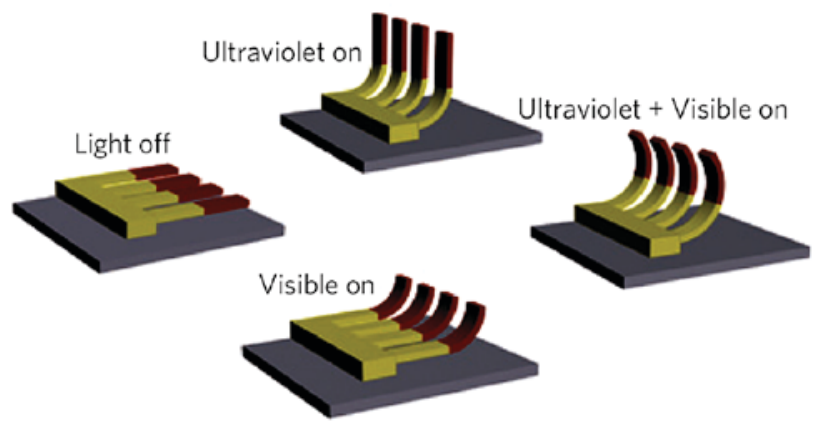

B

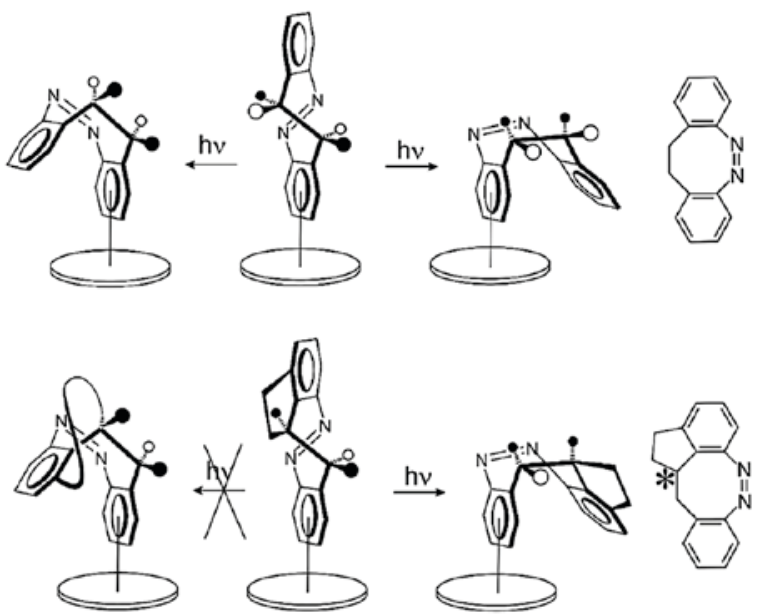

Figure 3. (A) Artificial, light-driven cilia produce an asymmetric motion controlled by the spectral composition of the light. Reproduced with permission (49). (B) Schematic representation of artificial cilia with diazocines as switching units. Reproduced with permission (33).
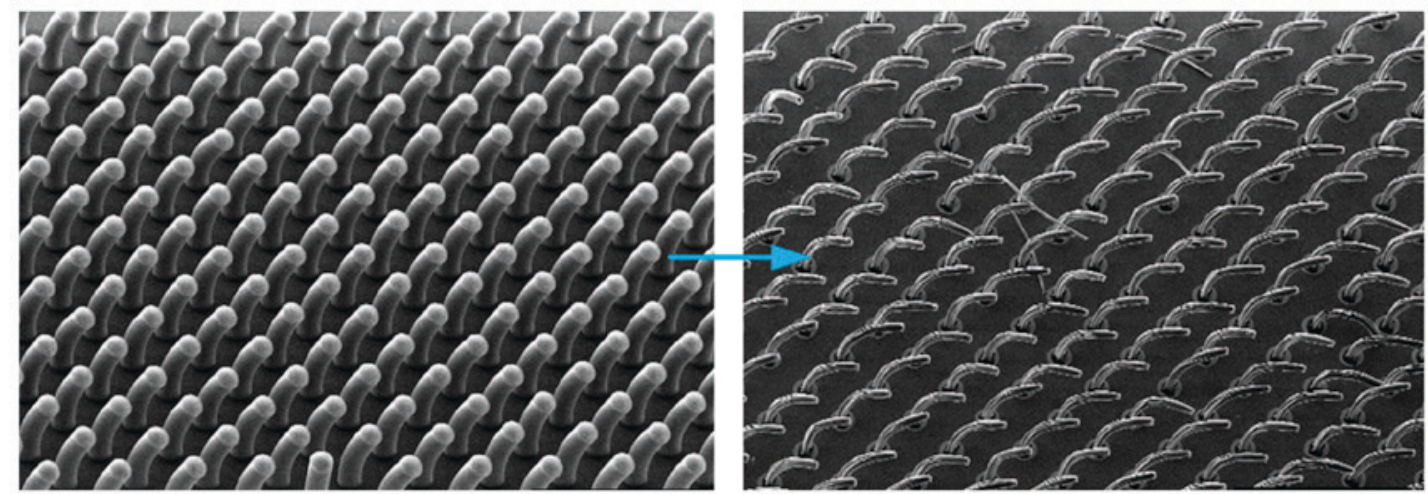

Figure 4. Scanning electron microscopy images of 3D carbon nanotubes cilium-like microstructures. Reproduced with permission (43).

accomplished progress of this motion occurred via special surface-containing cilia molecules, which concluded that a type molecular suction cup was being used to control the switch. This molecular suction cup may be adsorbed on the gold surface (49) successfully and maintains a flow state to attract the cilia molecules constantly following the complete self-assembly of cilia on the surface under light. Symmetric diazocines as well as asymmetric molecules were controlled to alter the directional motion of cilia (39). This study facilitated the understanding of artificial cilia, which as biological transport systems, are important in self-cleaning, thus providing a potential novel strategy for the treatment of diseases such as primary ciliary dyskinesia.

Force-actuated cilia. Force-actuated cilia are less widely researched because it is difficult to control cilium-directed motion; however, this kind of active cilia will potentially become the main type to be used in the engineering of artificial cilia in the future.

In previous years, researchers have provided novel ideas to solve the manufacturing problems of artificial cilia engineered mimicry, with increasing numbers of studies discussing carbon nanotubes (CNTs). A novel technique has been presented for scalable fabrication of freeform microstructures via stain-engineered growth of aligned CNTs (50).
This method represents a versatile principle for designing and manufacturing complex microstructured surfaces, as shown in Fig. 4. Four important problems surrounding CNTs require solving. Firstly, modulation of local CNT growth rate using offset patterning of the CNT growth catalyst. Secondly, confirmation of the final shape of curved CNT microstructures, designed via finite element modeling. Thirdly, overcoming the production of microstructures with multidirectional curvature and unusual self-organized patterns. Finally, the tuning of the mechanical properties independent from the microstructure geometry, by conformally coating CNTs.

In conclusion, the field of artificial cilia in bionic biology is still a relatively young field, and their implementation in commercial products is still rare. Nature has proven that the artificial cilia may be able to work in complex biological applications, due to their fluid manipulation effectiveness, their ability to locally control flows and the elegance of potential full integration, making them worthy for further investigation.

\section{Application of polymer brushes in the field of artificial cilia}

Polymer brushes have a similar topological structure to natural cilia. Although some size diversity exists, polymer brushes 


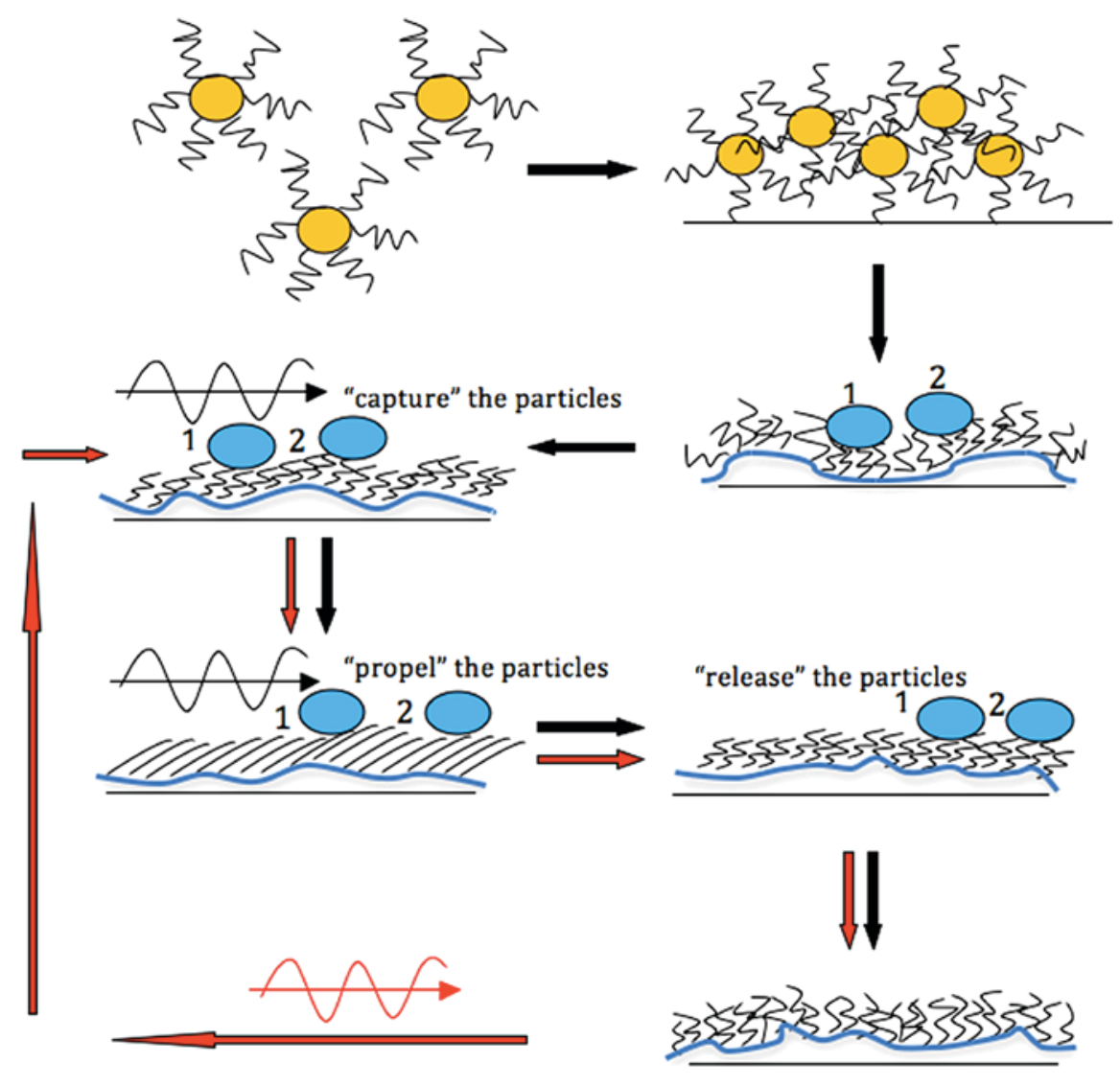

Figure 5. Schematic of polymer brush (yellow circles) filming following shaping, in order to remove attached matter (blue circles).

have had good applications in the field of surface modification, and additionally have various functions, including the delivery of medicine and genes $(51,52)$, enzyme biosynthesis $(53,54)$ and use in culture medium $(55,56)$. A recent study on artificial polymer brushes involved an artificial biological transportation system, as well as other research on a functional artificial epithelium, by applying an external stimulus on the carried nanoparticles to determine the effect of the brush as artificial nasal mucosa (39). Taken together, research into polymer brushes and cilia suggests that by combining polymer brushes and artificial cilium, a polymer net-structured material may be developed to imitate morphology and functionality of the natural cilium system. Proper design of the high polymer material and suitable polymer molding technology would be required. To achieve this design, the micromechanics of the polymer must be studied thoroughly.

At present, a polymer brush is treated as a fluid; thus, the rheology of polymer brushes is critical. This study of polymer brush rheology dates back to the 1950s (57), with discernable breakthroughs being been made in the 1970s (58). For polymer brushes, plenty of more recent studies point to polymer brush emulsion as a measure of its stability and rheological properties, based on Russel's (59) research, to develop the flow properties with polystyrene emulsion. These studies on the mechanical properties of the polymer revealed that the rheology is an important method in the characterization of these properties. Emulsion rheology has been extensively studied; however, there are limited studies on polymer brush film during the period of simulation. The main research has focused on variations of viscosity between each polymer brush layer under steady and oscillatory shear force. Alexander (60) conducted research on the absorption function of a flat sheet membrane with results demonstrating that stretching forms from free energy balance. On the basis of the free energy theory and experiment data, high-density polymer chains from the same layer overlap vertically, which may enhance the contact and interaction energy among chains. Polymer chains would be forced to stretch vertically from nodes, leading to a polymer density decrease, while the polymer brush thickness increases. Therefore, when solvents exist in a polymer brush system, the polymer brush stretches in order to obtain a larger contact area. On the other hand, when solvents do not exist in the system, polymer brushes stretch from the interface to avoid overlapping in the limited space. de Gennes (61) and Halperin et al (62) experimented on the self-assembly of the stratiform copolymer and its effect on the polymer chain, based on the model made by Alexander (60). They simulated the inner structure of the polymer brush based on their experiment data.

From the aspect of dynamics, mathematic models and calculations have been made to simulate the deformation of brush layers under force. Based on recent studies $(63,64)$, a few demonstrated that polymer brush layers incline when applied with shear force, but they are not sensitive to the magnitude of shear force. This property was observed particularly in the polymer brushes with nanoparticles embedded; their entropy stability makes it possible to maintain a stable performance under high shear force. Common research methods include 
the self-consistent field theory, the particle model and the coarse-grained model $(24,62,65)$. However, those methods may be only applied to a single component system; application to multiple component systems remains to be developed. As for a stimulus-response polymer brush, density functional theory is widely used to describe the structure deformation. Research has demonstrated that this may predict the thermodynamic and structural properties (24). In particular, description of a system's structural deformation prior to and following a stimulus made by dissipative particle dynamics would be vital to its practical use as well.

However, with its excellent ability of surface modification, polymer brush material following processing is applied in the field of biology as a kind of artificial cilia with a similar topology and suitable mechanical requirements in specific applications. For example, polymer brushes would be subject to constant shearing and washing in the inner face of the artificial trachea, brought about by airflow and mucus. This means that the polymer brush film must meet the shear test under certain conditions, so that the brush layer does not come off, flocculate or clump at a sufficient shear force. At the same time, the components of the mucus differ depending on the health and pathological states of the patient. Therefore, stretching side chains on the polymer brush film are required to be able to move various fluid environments.

Thus, it was hypothesized that the selection of appropriate monomers to create a polymer brush, as well as the coating material, with a similar ciliated topology, may be molded to help the artificial tracheal substitutes clear away airway wastes. The process is presented in Fig. 5. After shaping a cilia-like film layer, certain side chains of the polymer brush materials are adhesive to the base, and another part of the side chains intertwine with each other to form a film. Due to the steric hindrance, the rest of the side chains will extend into space freely. Meanwhile, the extending side chains of brush polymers end with a charge or some other features, including thermal, $\mathrm{pH}$ or any others in solution that will be able to generate electrostatic repulsion. Therefore, the spatial structure of the side chains is more extended, and may lead to increases in the height of the polymer film cilia-like layer. In addition, it may present many different properties when interacting with mucus.

In conclusion, progress has been made regarding using polymer brushes as a type of a surface modification of the epithelium following airway reconstruction, although further studies are required. Combined with the technology of polymer shaping, as well as making use of the advantages of polymer brushes to alter surface properties of the materials effectively, future studies may investigate coating films on the inner surface of reconstructed airways, which is produced with polymer brush material. These films may have similar topology to the natural epithelium and effectively replace the airway epithelial layer to clear away waste mucus. This has profound significance for the reconstruction of artificial trachea and the treatment of the tracheal defects.

\section{Acknowledgements}

This review was supported by the National Science Foundation of China (grant no. 31400805), the Chongqing
Natural Science Foundation (grant no. cstc2012jjA10069) and the Fundamental Research Funds for the Central Universities (grant no. CQDXWL-2013-026).

\section{References}

1. Atala A, Bauer SB, Soker S, Yoo JJ and Retik AB: Tissue-engineered autologous bladders for patients needing cystoplasty. Lancet 367: 1241-1246, 2006.

2. Jungebluth P, Alici E, Baiguera S, Le Blanc K, Blomberg P, Bozóky B, Crowley C, Einarsson O, Grinnemo KH, Gudbjartsson $\mathrm{T}$, et al: Tracheobronchial transplantation with a stem-cell-seeded bioartificial nanocomposite: A proof-of-concept study. Lancet 378: 1997-2004, 2011.

3. Omori k, Nakamura T, Kanemaru S, Asato R, Yamashita M, Tanaka S, Magrufov A, Ito J and Shimizu Y: Regenerative medicine of the trachea: The first human case. Ann Otol Rhinol Laryngol 114: 429-433, 2005.

4. Satoh S, Elstrodt J, Hinrichs WL, Feijen J and Wildevuur CR: Prevention of infection in a porous tracheal prosthesis by omental wrapping. ASAIO Trans 36: M438-M440, 1990.

5. Grillo HC: Tracheal replacement: A critical review. Ann Thorac Surg 73: 1995-2004, 2002.

6. Schultz P, Vautier D, Chluba J, Marcellin L and Debry C: Survival analysis of rats implanted with porous titanium tracheal prosthesis. Ann Thorac Surg 73: 1747-1751, 2002.

7. Liu HB, Yan Q, Wang C, Liu X, Wang C, Zhou XH and Xiao SJ: Saccharide- and temperature-responsive polymer brushes grown on gold nanoshells for controlled release of diols. Colloids and Surfaces A: Physicochemical and Engineering Aspects 386: 131-134, 2011.

8. Motornov M, Tam TK, Pita M, Tokarev I, Katz E and Minko S: Switchable selectivity for gating ion transport with mixed polyelectrolyte brushes: Approaching 'smart' drug delivery systems. Nanotechnology 20: 434006, 2009.

9. Raviv U, Giasson S, Kampf N, Gohy JF, Jérôme R and Klein J: Lubrication by charged polymers. Nature 425: 163-165, 2003.

10. Kobayashi M, Terada M and Takahara A: Polyelectrolyte brushes: A novel stable lubrication system in aqueous conditions. Faraday Discuss 156: 403-412; 413-434, 2012.

11. Tam TK, Pita M, Motornov M, Tokarev I, Minko S and Katz E: Modified electrodes with switchable selectivity for cationic and anionic redox species. Electroanalysis 22: 35-40, 2010.

12. Tam TK, Ornatska M, Pita M, Minko S and Katz E: Polymer brush-modified electrode with switchable and tunable redox activity for bioelectronic applications. J Phys Chem C 112: 8438-8445, 2008.

13. Dunderdale GJ, Howse JR and Fairclough JP: Controlling the motion and placement of micrometer-sized metal particles using patterned polymer brush surfaces. Langmuir 27: 11801-11805, 2011.

14. Dunderdale G, Howse J and Fairclough P: pH-dependent control of particle motion through surface interactions with patterned polymer brush surfaces. Langmuir 28: 12955-12961, 2012.

15. Yang X, Jiang Y, Shen B, Chen Y, Dong F, Yu K, Yang B and Lin Q: Thermo-responsive photoluminescent polymer brushes device as a platform for selective detection of $\mathrm{Cr}(\mathrm{VI})$. Polym Chem 4: 5591-5596, 2013.

16. Cammas S, Suzuki K, Sone C, Sakurai Y, Kataoka K and Okano T: Thermo-responsive polymer nanoparticles with a core-shell micelle structure as site-specific drug carriers. Journal of Controlled Release 48: 157-164, 1997.

17. Pinchasik BE, Tauer K, Möhwald H and Skirtach AG: Polymer brush gradients by adjusting the functional density through temperature gradient. Advanced Materials Interfaces 1: 1300056, 2014.

18. Yang T, Huh W, Kong H, Jho JY and Kim W II: Effects of polymer architecture and charge density on the $\mathrm{pH}$-responsive $\mathrm{Ca}(\mathrm{II})$ release from brushite. Colloids and Surfaces A: Physicochemical and Engineering Aspects 459: 74-81, 2014.

19. Xu Y, Chen X, Han X, Xu S, Liu H and Hu Y: Lock/unlock mechanism of solvent-responsive binary polymer brushes: Density functional theory approach. Langmuir 29: 4988-4997, 2013.

20. Yao K, Chen Y, Zhang J, Bunyard C and Tang C: Cationic salt-responsive bottle-brush polymers. Macromol Rapid Commun 34: 645-651, 2013.

21. Kaewsaiha P, Matsumoto K and Matsuoka H: Salt effect on the nanostructure of strong polyelectrolyte brushes in amphiphilic diblock copolymer monolayer on the water surface. Langmuir 23: 7065-7071, 2007. 
22. Moya SE, Azzaroni O, Kelby T, Donath E and Huck WT: Explanation for the apparent absence of collapse of polyelectrolyte brushes in the presence of bulky ions. J Phys Chem B 111: 7034-7040, 2007.

23. Cao QQ, Zuo CC and Li LJ: Molecular dynamics simulations of end-grafted centipede-like polymers with stiff charged side chains. The European Physical Journal E 32: 1-12, 2010.

24. Stuart MA, Huck WT, Genzer J, Müller M, Ober C, Stamm M, Sukhorukov GB, Szleifer I, Tsukruk VV, Urban M, et al: Emerging applications of stimuli-responsive polymer materials. Nat Mater 9: 101-113, 2010.

25. Luzinov I, Minko S and Tsukruk VV: Responsive brush layers: From tailored gradients to reversibly assembled nanoparticles. Soft Matter 4: 714-725, 2008

26. Xu C, Wu T, Drain CM, Batteas JD, Fasolka MJ and Beers KL: Effect of block length on solvent response of block copolymer brushes: Combinatorial study with block copolymer brush gradients. Macromolecules 39: 3359-3364, 2006.

27. Vogel G: News focus: Betting on cilia. Science 310: 216-218, 2005.

28. Jenkins PM, McEwen DP and Martens JR: Olfactory cilia: Linking sensory cilia function and human disease. Chem Senses 34: 451-464, 2009.

29. Fliegauf M, Benzing T and Omran H: When cilia go bad: Cilia defects and ciliopathies. Nat Rev Mol Cell Biol 8: 880-893, 2007.

30. Ainsworth C: Cilia: Tails of the unexpected. Nature 448 : 638-641, 2007

31. den Toonder JM and Onck PR: Microfluidic manipulation with artificial/bioinspired cilia. Trends Biotechnol 31: 85-91, 2013.

32. Vilfan M, Potocnik A, Kavcic B, Osterman N, Poberaj I, Vilfan A and Babic D: Self-assembled artificial cilia. Proc Natl Acad Sci USA 107: 1844-1847, 2010

33. Toonder Jd, Bos F, Broer D, Filippini L, Gillies M, de Goede J, Mol T, Reijme M, Talen W, Wilderbeek H, et al: Artificial cilia for active micro-fluidic mixing. Lab Chip 8: 533-541, 2008

34. Khatavkar VV, Anderson PD, den Toonder JMJ and Meijer HEH Active micromixer based on artificial cilia. Physics Fluids 19 $083605,2007$.

35. Fahrni F, Prins MW and van Ijzendoorn LJ: Micro-fluidic actuation using magnetic artificial cilia. Lab Chip 9: 3413-3421, 2009.

36. Khaderi SN, Craus CB, Hussong J, Schorr N, Belardi J, Westerweel J, Prucker O, Rühe J, den Toonder JM and Onck PR: Magnetically-actuated artificial cilia for microfluidic propulsion Lab Chip 11: 2002-2010, 2011.

37. Hussong J, Schorr N, Belardi J, Prucker O, Rühe J and Westerweel J: Experimental investigation of the flow induced by artificial cilia. Lab Chip 11: 2017-2022, 2011

38. van Oosten CL, Bastiaansen CW and Broer DJ: Printed artificial cilia from liquid-crystal network actuators modularly driven by light. Nat Mater 8: 677-682, 2009.

39. Tellkamp T, Shen J, Okamoto Y and Herges R: Diazocines on molecular platforms. Eur J Org Chem 25: 5456-5461, 2014.

40. Zarzar LD, Kim P and Aizenberg J: Bio-inspired design of submerged hydrogel-actuated polymer microstructures operating in response to $\mathrm{pH}$. Adv Mater 23: 1442-1446, 2011.

41. Liu F, Ramachandran D and Urban MW: Colloidal films that mimic cilia. Adv Functional Materials 20: 3163-3167, 2010.

42. Liu F and Urban MW: Dual temperature and $\mathrm{pH}$ responsiveness of poly(2-(N,N-dimethylamino)ethyl methacrylate-co-n-butyl acrylate) colloidal dispersions and their films. Macromolecules 41 : 6531-6539, 2008

43. Liu F, Jarrett WL and Urban MW: Glass (Tg) and stimuli-responsive $\left(\mathrm{T}_{\mathrm{SR}}\right)$ transitions in random copolymers. Marcomolecules 43 : $5330-5337,2010$

44. Liu F and Urban MW: 3D directional temperature responsive (N-(DL)-(1-Hydroxymethyl) propylmethacrylamide-co-n-butyl Acrylate) colloids and their coalescence. Macromolecules 41 : 352-360, 2008
45. Khaderi S, Hussong J, Westerweel J, den Toonder J and Onck P: Fluid propulsion using magnetically-actuated artificial cilia-experiments and simulations. RSC Adv 3: 12735-12742, 2013.

46. Breidenich JL, Wei MC, Clatterbaugh GV, Benkoski JJ, Keng PY and Pyun J: Controlling length and areal density of artificial cilia through the dipolar assembly of ferromagnetic nanoparticles. Soft Matter 8: 5334-5341, 2012.

47. Khaderi SN, den Toonder JM and Onck PR: Magnetically actuated artificial cilia: The effect of fluid inertia. Langmuir 28: 7921-7937, 2012.

48. Evans BA, Shields AR, Carroll RL, Washburn S, Falvo MR and Superfine R: Magnetically actuated nanorod arrays as biomimetic cilia. Nano Lett 7: 1428-1434, 2007.

49. Klajn R: Immobilized azobenzenes for the construction of photoresponsive materials. Pure Appl Chem 82: 2247-2279, 2010.

50. De Volder M, Park S, Tawfick S and Hart AJ: Stain-engineered manufacturing of freeform carbon nanotube microstructures. Nat Commun 5: 4512, 2014.

51. Cammas S, Suzuki K, Sone C, Sakurai Y, Kataoka K and Okano T: Thermo-responsive polymer nanoparticles with a core-shell micelle structure as site-specific drug carriers. J Controlled Release 48: 157-164, 1997.

52. Alvarez-Lorenzo C and Concheiro A: Molecularly imprinted polymers for drug delivery. J Chromatography B 804: 231-245, 2004.

53. Zhu QZ, Liu F, Li DH, Xu JG, Su W and Huang J: A novel polymer-mimetic enzyme immunoassay system based on thermal phase separating technique. Analytica Chimica Acta 375: 177-185, 1998.

54. Matsukata M, Aoki T, Sanui K, Ogata N, Kikuchi A, Sakurai Y and Okano T: Effect of molecular architecture of poly(N-isoprop ylacrylamide)-trypsin conjugates on their solution and enzymatic properties. Bioconjug Chem 7: 96-101, 1996.

55. Yamada N, Okano T, Sakai H, Karikusa F, Sawasaki Y and Sakurai Y: Thermo-responsive polymeric surfaces; control of attachment and detachment of cultured cells. Macromolecular Rapid Commun 11: 571-576, 1990.

56. Mizutani A, Kikuchi A, Yamato M, Kanazawa H and Okano T: Preparation of thermoresponsive polymer brush surfaces and their interaction with cells. Biomaterials 29: 2073-2081, 2008.

57. Yuan LW: Rheology Theory. Shanghai Scientific and Technical Publishers, Shanghai, 1961 (In Chinese).

58. Qian XS: The Modern Mechanics - The Speech at the Meeting of the Nationwide Mechanical Design in 1978. Mechanics in Engineering 1: 4-9, 1979 (In Chinese).

59. Russel WB, Saville DA and Schowalter WR (eds): Colloidal Dispersions. Cambridge University Press, Cambridge, 1989.

60. Alexander S: Adsorption of chain molecules with a polar head a scaling description. J Phys France 38: 983-987, 1977.

61. de Gennes PG: Conformations of polymers attached to an interface. Macromolecules 13: 1069-1075, 1980.

62. Halperin A, Tirrell M and Lodge TP: Tethered chains in polymer microstructures. Adv Polymer Sci 100: 31-71, 1992.

63. Milchev A, Dimitrov DI and Binder K: Polymer brushes with nanoinclusions under shear: A molecular dynamics investigation. Biomicrofluidics 4: 32202, 2010.

64. An S, Choi SK, Cho JW, Kim HT and Kim JW: Colloidal interactions of inorganic nanoparticles Grafted with zwitterionic polymer brushes and gels by surface-mediated seeded polymerization. Macromol Rapid Commun 35: 1356-1361, 2014.

65. de Gennes PG: Conformations of polymers attached to an interface. Macromolecules 13: 1069-1075, 1980. 